\title{
Adams' type inequality and application to a quasilinear nonhomogeneous equation with singular and vanishing radial potentials in $\mathbb{R}^{4}$
}

\author{
Sami Aouaoui ${ }^{1}$ (D) Francisco S. B. Albuquerque ${ }^{2}$
}

Received: 3 April 2018 / Accepted: 22 December 2018 / Published online: 4 January 2019

(c) Fondazione Annali di Matematica Pura ed Applicata and Springer-Verlag GmbH Germany, part of Springer Nature 2019

\begin{abstract}
In this paper, we establish some Adams' type inequality for weighted second-order Sobolev spaces in four dimensions. The weights are radial and can have a singular or decaying behavior. This inequality is used to study some nonhomogeneous quasilinear elliptic equation.
\end{abstract}

Keywords Adams' inequality · Singular or decaying weights · Radial functions ·

Nonhomogeneous quasilinear elliptic equation $\cdot$ Exponential critical growth

Mathematics Subject Classification 35A23 · 35B33 · 35J30 · 35J35 · 35J91

\section{Introduction and main result}

Let $\Omega$ be a bounded domain in $\mathbb{R}^{N}, N \geq 2$. The famous classical Trudinger-Moser inequality for the limiting case of Sobolev inequalities (see [25,36]) states that, for all $u \in W_{0}^{1, N}(\Omega)$ and $\alpha>0$, we have

$$
\sup _{|\nabla u|_{N} \leq 1} \int_{\Omega} e^{\alpha|u|^{N-1}} \mathrm{~d} x\left\{\begin{array}{l}
<+\infty, \\
=+\infty,
\end{array}\right.
$$

where $\alpha_{N}=N \omega_{N-1}^{\frac{1}{N-1}},|\nabla u|_{N}$ is the Dirichlet norm, that is, $|\nabla u|_{N}:=\left(\int_{\Omega}|\nabla u|^{N} \mathrm{~d} x\right)^{\frac{1}{N}}$ and $\omega_{N-1}$ is the measure of the unit sphere in $\mathbb{R}^{N}$. In addition, for any bounded domain $\Omega$, the supremum in (1.1) can be attained. This result has been generalized and extended in many

Sami Aouaoui

sami.aouaoui@ismai.u-kairouan.tn

Francisco S. B. Albuquerque

fsiberio@cct.uepb.edu.br

1 Institut Supérieur des Mathématiques Appliquées et de l'Informatique de Kairouan, Avenue Assad Iben Fourat, 3100 Kairouan, Tunisia

2 Departamento de Matemática, Universidade Estadual da Paraíba, CEP 58429-500 Campina Grande, PB, Brazil 
directions in recent years. For example, some extended versions of the Trudinger-Moser inequality to unbounded domains in Euclidean spaces were established in [1,10,12,13,22,28].

The Adams' inequality is a natural extension of the Trudinger-Moser inequality for the higher-order derivatives. In 1988, Adams [2] established the following result: For $m \in \mathbb{N}$ and $\Omega$ an open bounded set of $\mathbb{R}^{N}$ such that $m<N$, there exists a positive constant $C_{m, N}$ such that

$$
\sup _{u \in W_{0}^{m, \frac{N}{m}}(\Omega),\left|\nabla^{m} u\right|_{\frac{N}{m} \leq 1}} \int_{\Omega} e^{\beta_{0}|u|^{\frac{N}{N-m}}} \mathrm{~d} x \leq C_{m, N}|\Omega|,
$$

where

$$
\beta_{0}=\beta_{0}(m, N):=\frac{N}{\omega_{N-1}} \begin{cases}{\left[\frac{\pi^{\frac{N}{2}} 2^{m} \Gamma\left(\frac{m}{2}\right)}{\Gamma\left(\frac{N-m}{2}\right)}\right]^{\frac{N}{N-m}},} & \text { if } \mathrm{m} \text { is even } \\ {\left[\frac{\pi^{\frac{N}{2}} 2^{m} \Gamma\left(\frac{m+1}{2}\right)}{\Gamma\left(\frac{N-m+1}{2}\right)}\right]^{\frac{N}{N-m}},} & \text { if } \mathrm{m} \text { is odd }\end{cases}
$$

and

$$
\nabla^{m} u:= \begin{cases}\Delta^{\frac{m}{2}} u, & \mathrm{~m} \text { even } \\ \nabla \Delta^{\frac{m-1}{2}} u, & \mathrm{~m} \text { odd }\end{cases}
$$

denotes the $m$ th-order gradient of $u$. Furthermore, inequality (1.2) is sharp, that is, for any $\beta>\beta_{0}$, the integral in (1.2) can be made as large as possible.

Plainly, the Adams' inequality in its form (1.2) cannot be extended to unbounded domains. For $t \geq 0$, set

$$
\phi(t)=e^{t}-\sum_{j=0}^{j_{\frac{N}{m}}-2} \frac{t^{j}}{j !}, \quad m \in \mathbb{N}, \quad N>m, j_{\frac{N}{m}}=\min \left\{j \in \mathbb{N}, \quad j \geq \frac{N}{m}\right\} .
$$

A first attempt to generalize Adams' inequality to unbounded domains is due to Ozawa who, in [26], proved the existence of two positive constants $\alpha$ and $C$ such that

$$
\int_{\mathbb{R}^{N}} \phi\left(\alpha|u|^{\frac{N}{N-m}}\right) \mathrm{d} x \leq C|u|_{\frac{N}{m}}^{\frac{N}{m}}, \forall u \in W^{m, \frac{N}{m}}\left(\mathbb{R}^{N}\right),\left|\nabla^{m} u\right|_{\frac{N}{m}} \leq 1 .
$$

In the particular case (which is the most interesting in our present work) where $N=4$ and $m=2$, inequality (1.3) takes the form

$$
\int_{\mathbb{R}^{4}}\left(e^{\alpha u^{2}}-1\right) \mathrm{d} x \leq C|u|_{2}^{2}, \forall u \in H^{2}\left(\mathbb{R}^{4}\right),|\Delta u|_{2} \leq 1 .
$$

In 2013, Ruf and Sani [29] established the following very useful result: Let $m=2 k$ an even integer less than $N$. Then, there exists a constant $C_{m, N}>0$ such that, for any domain $\Omega \subset \mathbb{R}^{N}$, we have

$$
\sup _{u \in W_{0}^{m, \frac{N}{m}}(\Omega),\left|(-\Delta+I)^{k} u\right|_{\frac{N}{m}} \leq 1} \int_{\Omega} \phi\left(\beta_{0}|u|^{\frac{N}{N-m}}\right) \mathrm{d} x \leq C_{m, N},
$$


and this inequality is sharp. In the particular case where $N=4, m=2$ and $\Omega=\mathbb{R}^{4}$, inequality (1.5) becomes

$$
\sup _{u \in H^{2}\left(\mathbb{R}^{4}\right),|(-\Delta+I) u|_{2} \leq 1} \int_{\mathbb{R}^{4}}\left(e^{32 \pi^{2} u^{2}}-1\right) \mathrm{d} x<+\infty .
$$

Many other improvements of the Adams' inequality in bounded and unbounded domains have been proved. We can refer to [17,19,23,24,35,37]. Among them, we can cite the following inequality, proved by Masmoudi and Sani [24]:

$$
\int_{\mathbb{R}^{4}} \frac{e^{32 \pi^{2} u^{2}}-1}{(1+|u|)^{2}} \mathrm{~d} x \leq C|u|_{2}^{2}, \forall u \in H^{2}\left(\mathbb{R}^{4}\right),|\Delta u|_{2} \leq 1 .
$$

Moreover, (1.7) is no longer true if the exponent 2 is replaced by a real number $p<2$. Furthermore, as it was mentioned in [24], inequalities (1.6) and (1.3) can be derived from (1.7). Another improvement of Adams' inequality (1.2) in bounded domains has been provided by Tarsi [35]. In fact, in [35], the author obtained some interesting embeddings in Zygmund spaces. One result of these embeddings is the following stronger version of Adams' inequality (1.2) in the particular case where $m=2$ and $N=4$ : Let $\Omega$ a bounded domain of $\mathbb{R}^{4}$, then there exists a constant $C>0$ such that

$$
\sup _{u \in H^{2}(\Omega) \cap H_{0}^{1}(\Omega),|\Delta u|_{2} \leq 1} \int_{\Omega} e^{32 \pi^{2} u^{2}} \mathrm{~d} x \leq C|\Omega|,
$$

and this inequality is also sharp. Observing that $H_{0}^{2}(\Omega)$ is strictly contained in $H^{2}(\Omega) \cap$ $H_{0}^{1}(\Omega)$, we can see that (1.8) shows that the optimal exponent $32 \pi^{2}$ depends only on the first-order trace. Now, concerning the singular case, by introducing singular weights of the form $\frac{1}{|x|^{\alpha}}$, we have the following inequality proved by Lam and Lu [17] which will be of big interest in our work: Let $0 \leq \alpha<4$ and $\Omega$ be a bounded domain in $\mathbb{R}^{4}$. Then, for all $0 \leq \beta \leq 32 \pi^{2}\left(1-\frac{\alpha}{4}\right)$, we have

$$
\sup _{u \in H^{2}(\Omega) \cap H_{0}^{1}(\Omega),|\Delta u|_{2} \leq 1} \int_{\Omega} \frac{e^{\beta u^{2}}}{|x|^{\alpha}} \mathrm{d} x<+\infty .
$$

When $\beta>32 \pi^{2}\left(1-\frac{\alpha}{4}\right)$, the supremum is infinite.

Our present work can be considered as a contribution in this direction. In fact, the main purpose of this paper is to establish a new inequality of Adams' type where some radial weights that can be singular at the origin, unbounded or vanishing at infinity are involved and we will apply it to study some elliptic quasilinear equation by using a variational framework based on a nonstandard Orlicz space. Here, we have to highlight the fact that our results concern radial functions.

In a more precise way and throughout this work, we consider some radial weight functions $V(|x|)$ and $K(|x|)$ satisfying the following assumptions:

$(V) V:(0, \infty) \rightarrow \mathbb{R}$ is continuous, $V(r)>0$ for all $r>0$ and there exist $a_{0}>-4, a>-6$ such that

$$
\limsup _{r \rightarrow 0^{+}} \frac{V(r)}{r^{a_{0}}}<+\infty \text { and } \liminf _{r \rightarrow+\infty} \frac{V(r)}{r^{a}}>0
$$


$(K) K:(0, \infty) \rightarrow \mathbb{R}$ is continuous, $K(r)>0$ for all $r>0$ and there exist $b_{0}>-4, b<a$ such that

$$
\limsup _{r \rightarrow 0^{+}} \frac{K(r)}{r^{b_{0}}}<\infty \text { and } \limsup _{r \rightarrow+\infty} \frac{K(r)}{r^{b}}<\infty .
$$

This kind of weight functions was firstly introduced by Su et al. [33] (see also [34]) in the study of a nonlinear Schrödinger equation. In [33], the authors studied the existence of solution for the problem

$$
\left\{\begin{array}{l}
-\Delta u+V(|x|) u=K(|x|) f(u), \\
x \in \mathbb{R}^{N} \\
|u(x)| \rightarrow 0 \text { as }|x| \rightarrow \infty
\end{array}\right.
$$

where the nonlinearity considered was $f(s)=|s|^{q-2} s$, with $2<q<2^{*}=\frac{2 N}{N-2}$ for $N \geq 3$ and $2<q<\infty$ if $N=2$. Succeeding this study, Albuquerque et al. [4] studied the above problem in the critical case suggested by Trudinger-Moser inequality (1.1). The above restrictions on $V$ and $K$ describing the behavior of the weights near the origin and infinity are crucial to obtain our embedding result (see Lemma 2.3) as well as Adams' inequality (see Theorem 1.2).

Example 1.1 1. Typical models of weights $V$ and $K$ satisfying $(V)-(K)$, respectively, can be found, for instance, in $[3,4,6]$, and they are of the form

$$
V(r)=\left\{\begin{array}{ll}
r^{\gamma_{1}}, & \text { if } r \leq 1, \\
r^{\gamma_{2}}, & \text { if } r \geq 1,
\end{array} \text { and } K(r)=r^{\gamma_{3}}\right.
$$

with $\gamma_{1}>-4, \gamma_{2}>-4$ and $-4<\gamma_{3}<\gamma_{2}$.

$$
\frac{A_{1}}{1+r^{\gamma_{1}}} \leq V(r) \leq A_{2} \text { and } 0<K(r) \leq \frac{A_{3}}{1+r^{\gamma_{2}}}
$$

for positive constants $A_{1}, A_{2}, A_{3}$, with $\gamma_{1} \in(0,6)$ and $\gamma_{2}>\gamma_{1}$.

2. One can also take

$$
V(r)=\frac{3+\cos r}{r} \text { and } K(r)=\frac{1+e^{-r^{2}} \sin r}{3 r^{\frac{5}{2}}} .
$$

Clearly, if we take $a=a_{0}=-1$ and $b=b_{0}=-\frac{5}{2}$, then $V$ and $K$ are singular at the origin and vanishing at infinity.

In order to state our results, we need to introduce some notations. If $1 \leq q<\infty$, we define the weighted Lebesgue spaces

$$
L^{q}\left(\mathbb{R}^{4} ; K\right):=\left\{u: \mathbb{R}^{4} \rightarrow \mathbb{R}, u \text { is measurable and } \int_{\mathbb{R}^{4}} K(|x|)|u|^{q} \mathrm{~d} x<\infty\right\}
$$

and

$$
L^{q}\left(\mathbb{R}^{4} ; V\right):=\left\{u: \mathbb{R}^{4} \rightarrow \mathbb{R}, u \text { is measurable and } \int_{\mathbb{R}^{4}} V(|x|)|u|^{q} \mathrm{~d} x<\infty\right\},
$$


endowed, respectively, with the norms

$$
|u|_{L^{q}\left(\mathbb{R}^{4} ; K\right)}=\left(\int_{\mathbb{R}^{4}} K(|x|)|u|^{q} \mathrm{~d} x\right)^{\frac{1}{q}} \text { and }|u|_{L^{q}\left(\mathbb{R}^{4} ; V\right)}=\left(\int_{\mathbb{R}^{4}} V(|x|)|u|^{q} \mathrm{~d} x\right)^{\frac{1}{q}} .
$$

Let $C_{0}^{\infty}\left(\mathbb{R}^{4}\right)$ be the set of smooth functions with compact support and $D^{1,2}\left(\mathbb{R}^{4}\right)$ the closure of $C_{0}^{\infty}\left(\mathbb{R}^{4}\right)$ under the norm

$$
|\nabla u|_{2}=\left(\int_{\mathbb{R}^{4}}|\nabla u|^{2} \mathrm{~d} x\right)^{\frac{1}{2}}
$$

Observe that since $4>2$, then one can define $D^{1,2}\left(\mathbb{R}^{4}\right)$ as follows

$$
D^{1,2}\left(\mathbb{R}^{4}\right)=\left\{u \in L_{\text {loc }}^{1}\left(\mathbb{R}^{4}\right),|\nabla u| \in L^{2}\left(\mathbb{R}^{4}\right)\right\} .
$$

Denote by $D_{\text {rad }}^{1,2}\left(\mathbb{R}^{4}\right)$ the subspace of the radial functions in $D^{1,2}\left(\mathbb{R}^{4}\right)$. Set

$$
E:=\left\{u \in D_{\mathrm{rad}}^{1,2}\left(\mathbb{R}^{4}\right) \cap L^{2}\left(\mathbb{R}^{4} ; V\right), \Delta u \in L^{2}\left(\mathbb{R}^{4}\right)\right\},
$$

which is a Hilbert space endowed with the inner product

$$
\langle u, v\rangle:=\int_{\mathbb{R}^{4}}[\Delta u \Delta v+\nabla u \nabla v+V(|x|) u v] \mathrm{d} x, \quad u, v \in E,
$$

and the corresponding norm given by

$$
\|u\|:=\left(\int_{\mathbb{R}^{4}}\left[|\Delta u|^{2}+|\nabla u|^{2}+V(|x|) u^{2}\right] \mathrm{d} x\right)^{\frac{1}{2}}, \quad u \in E .
$$

Our Adams' type inequality is stated in the following theorem.

Theorem 1.2 Assume that $(V)-(K)$ hold. Then, for any $u \in E$ and $\alpha>0$, we have that $K(|x|)\left(e^{\alpha u^{2}}-1\right) \in L^{1}\left(\mathbb{R}^{4}\right)$. Furthermore, if $\alpha \leq \alpha_{0}:=\min \left\{32 \pi^{2}, 8 \pi^{2}\left(4+b_{0}\right)\right\}$, there holds

$$
\sup _{u \in E,\|u\| \leq 1} \int_{\mathbb{R}^{4}} K(|x|)\left(e^{\alpha u^{2}}-1\right) \mathrm{d} x<\infty
$$

Moreover, in the case where $-4<b_{0} \leq 0$, if we also assume that $\liminf _{r \rightarrow 0^{+}} \frac{K(r)}{r^{b_{0}}}>0$, then the value $\alpha_{0}$ is optimal, that is,

$$
\sup _{u \in E,\|u\| \leq 1} \int_{\mathbb{R}^{4}} K(|x|)\left(e^{\alpha u^{2}}-1\right) \mathrm{d} x=+\infty, \quad \forall \alpha>\alpha_{0} .
$$

The outline of the paper is as follows In the forthcoming section, we establish some embedding results involving appropriate weighted Lebesgue spaces. In Sect. 3, we prove our main result, Theorem 1.2. Section 4 is devoted to study a nonhomogeneous quasilinear elliptic problem using our Adams' type inequality.

Throughout this paper, we use $|\cdot|_{p}$ to denote the norm of the Lebesgue space $L^{p}\left(\mathbb{R}^{4}\right), 1 \leq$ $p \leq \infty$, and the symbols $C, C_{0}, C_{1}, C_{2}, \ldots$ to denote positive generic constants (possibly different). 


\section{Embedding results: compactness}

In the following, we denote by $B(x, R) \subset \mathbb{R}^{4}$ the open ball centered at $x \in \mathbb{R}^{4}$ with radius $R>0$ and, to simplify notations, we set $B_{R}:=B(0, R), B_{R}^{c}:=\mathbb{R}^{4} \backslash B_{R}$ and $B_{R} \backslash B_{r}$ denotes the annulus with interior radius $r$ and exterior radius $R$. Here is some new variant of the well-known radial Lemma of Strauss [32].

Lemma 2.1 Assume that $(V)$ holds. Then, there exist $R_{0}>0$ and $C>0$ such that for all $u \in E$,

$$
|u(x)| \leq C\|u\||x|^{-(a+6) / 4}, \quad|x|>R_{0} .
$$

Proof The proof follows some arguments developed in [4,33,34]. For the convenience of the reader, we give it in detail. By a standard density argument, it suffices to prove (2.1) for radial functions $u \in C_{0}^{\infty}\left(\mathbb{R}^{4}\right)$. Let $\rho=|x|$ and $\varphi:[0,+\infty) \rightarrow \mathbb{R}$ be such that $\varphi(\rho)=u(|x|)$. Since $a>-6$, one has

$$
\frac{\mathrm{d}}{\mathrm{d} \rho}\left[\rho^{(a+6) / 2} \varphi^{2}(\rho)\right]=\frac{a+6}{2} \rho^{(a+4) / 2} \varphi^{2}(\rho)+2 \rho^{(a+6) / 2} \varphi(\rho) \varphi^{\prime}(\rho) \geq 2 \rho^{(a+6) / 2} \varphi(\rho) \varphi^{\prime}(\rho) .
$$

It follows from $(V)$ that there exist $R_{0}>0$ and $C_{0}>0$ such that

$$
V(|x|) \geq C_{0}|x|^{a}, \quad \text { for all }|x| \geq R_{0} .
$$

Then for $\rho>R_{0}$, by (2.2), (2.3) and the Hölder's inequality, it follows

$$
\begin{aligned}
\rho^{(a+6) / 2} \varphi^{2}(\rho) & \leq 2 \int_{\rho}^{\infty} s^{(a+6) / 2}|\varphi(s)|\left|\varphi^{\prime}(s)\right| \mathrm{d} s=2 \int_{\rho}^{\infty}\left(\left|\varphi^{\prime}(s)\right| s^{3 / 2}\right)\left(s^{a / 2}|\varphi(s)| s^{3 / 2}\right) \mathrm{d} s \\
& \leq 2\left(\int_{\rho}^{\infty}\left|\varphi^{\prime}(s)\right|^{2} s^{3} \mathrm{~d} s\right)^{1 / 2}\left(\int_{\rho}^{\infty} s^{a}|\varphi(s)|^{2} s^{3} \mathrm{~d} s\right)^{1 / 2} \\
& \leq C\left(\int_{B_{\rho}^{c}}|\nabla u|^{2} \mathrm{~d} x\right)^{1 / 2}\left(\int_{B_{\rho}^{c}} V(|x|) u^{2} \mathrm{~d} x\right)^{1 / 2} \leq C\|u\|^{2} .
\end{aligned}
$$

Thus, we conclude that $|u(x)| \leq C\|u\||x|^{-(a+6) / 4}$, for all $|x|>R_{0}$, which completes the proof.

Remark 2.2 One can easily observe that in the last proof, we have proved a more precise result than the one stated in Lemma 2.1. In reality, we prove the existence of two positive constants $C$ and $R_{0}$ such that

$$
|u(x)| \leq C|x|^{-\frac{a+6}{4}} \mathcal{V}(u), \quad \forall|x|>R_{0},
$$

where

$$
\mathcal{V}(u)=\left(\int_{\mathbb{R}^{4}}|\nabla u|^{2} \mathrm{~d} x+\int_{\mathbb{R}^{4}} V(|x|) u^{2} \mathrm{~d} x\right)^{\frac{1}{2}} .
$$

Now, using Lemma 2.1 and taking profit of some results established in [33] (see also [4]), we have the following embedding result which will be used in the sequel of the paper.

Lemma 2.3 Suppose that $(V)$ and $(K)$ hold. Then, the space $E$ is continuously and compactly immersed in $L^{q}\left(\mathbb{R}^{4} ; K\right)$ for each $2 \leq q<\infty$. 
Proof For $q \geq 2$, consider the quantity $S_{q}:=\inf _{u \in E \backslash\{0\}} \frac{\|u\|^{2}}{|u|_{L^{q}\left(\mathbb{R}^{4} ; K\right)}^{2}}$. To prove the continuity of the embedding $E \hookrightarrow L^{q}\left(\mathbb{R}^{4} ; K\right)$, it suffices to show that $S_{q}>0$. Suppose the contrary, that is, $S_{q}=0$. Then, there exists a sequence $\left(u_{n}\right) \subset E$ satisfying

$$
\left|u_{n}\right|_{L^{q}\left(\mathbb{R}^{4} ; K\right)}=1 \text { and } \lim _{n \rightarrow \infty}\left\|u_{n}\right\|^{2}=0 .
$$

By the hypothesis $(K)$, there exists a positive constant $C_{1}$ such that

$$
K(|x|) \leq C_{1}|x|^{b}, \text { for all }|x| \geq R_{0} .
$$

Now, for $R>R_{0}$, by Lemma 2.1, (2.3) and (2.4), one has

$$
\begin{aligned}
\int_{B_{R}^{c}} K(|x|)\left|u_{n}\right|^{q} \mathrm{~d} x & \leq C_{1} \int_{B_{R}^{c}}|x|^{b}\left|u_{n}\right|^{q} \mathrm{~d} x=C_{1} \int_{B_{R}^{c}}|x|^{b-a}\left|u_{n}\right|^{q-2}|x|^{a} u_{n}^{2} \mathrm{~d} x \\
& \leq C_{2}\left\|u_{n}\right\|^{q-2} \int_{B_{R}^{c}}|x|^{b-a-(q-2) \frac{a+6}{4}} V(|x|) u_{n}^{2} \mathrm{~d} x .
\end{aligned}
$$

Since $a>-6, b<a$ and $q \geq 2$, then $b-a-\frac{(q-2)(a+6)}{4}<0$. Thus, we obtain

$$
\int_{B_{R}^{c}} K(|x|)\left|u_{n}\right|^{q} \mathrm{~d} x \leq C_{2} R^{b-a-(q-2) \frac{a+6}{4}}\left\|u_{n}\right\|^{q} .
$$

Again due to $(K)$, there exist $0<r_{0}<1 / 2$ and $C_{3}>0$ such that

$$
K(|x|) \leq C_{3}|x|^{b_{0}}, \quad \text { for all } 0<|x|<r_{0} .
$$

Now, we shall estimate the integral $\int_{B_{r_{0}}} K(|x|)\left|u_{n}\right|^{q} \mathrm{~d} x$. For that aim, consider $\sigma>1$ be such that $b_{0} \sigma>-4$ and define a radial cutoff function $\psi \in C_{0}^{\infty}\left(B_{1}\right)$ verifying $0 \leq \psi \leq 1$ in $B_{1}, \psi \equiv 1$ in $B_{1 / 2}, \psi \equiv 0$ in $B_{1} \backslash B_{3 / 4}$ and $\max \{|\nabla \psi|,|\Delta \psi|\} \leq C$ in $B_{1}$.

By (2.6), Hölder's inequality, and the continuous embedding $H_{0}^{2}\left(B_{1}\right) \hookrightarrow L^{\frac{q \sigma}{\sigma-1}}\left(B_{1}\right)$, we have

$$
\begin{aligned}
& \int_{B_{r_{0}}} K(|x|)\left|u_{n}\right|^{q} \mathrm{~d} x \leq C_{3} \int_{B_{r_{0}}}|x|^{b_{0}}\left|u_{n}\right|^{q} \mathrm{~d} x=C_{3} \int_{B_{r_{0}}}|x|^{b_{0}}\left|\psi u_{n}\right|^{q} \mathrm{~d} x \\
& \leq C_{3}\left(\int_{B_{r_{0}}}|x|^{b_{0} \sigma} \mathrm{d} x\right)^{1 / \sigma}\left(\int_{B_{r_{0}}}\left|\psi u_{n}\right|^{\frac{q \sigma}{\sigma-1}} \mathrm{~d} x\right)^{\frac{\sigma-1}{\sigma}} \\
& \leq C_{4} r_{0}^{b_{0}+\frac{4}{\sigma}}\left(\int_{B_{1}}\left|\psi u_{n}\right|^{\frac{q \sigma}{\sigma-1}} \mathrm{~d} x\right)^{\frac{\sigma-1}{\sigma}} \leq C_{5} r_{0}^{b_{0}+\frac{4}{\sigma}}\left(\int_{B_{1}}\left|\Delta\left(\psi u_{n}\right)\right|^{2} \mathrm{~d} x\right)^{\frac{q}{2}} \\
& \leq C_{6} r_{0}^{b_{0}+\frac{4}{\sigma}}\left(\int_{B_{1}}\left|u_{n} \Delta \psi\right|^{2} \mathrm{~d} x+\int_{B_{1}}\left|\nabla \psi \cdot \nabla u_{n}\right|^{2} \mathrm{~d} x+\int_{B_{1}}\left|\psi \Delta u_{n}\right|^{2} \mathrm{~d} x\right)^{\frac{q}{2}} \\
& \leq C_{7} r_{0}^{b_{0}+\frac{4}{\sigma}}\left(\int_{B_{3 / 4} \backslash B_{1 / 2}}\left|u_{n}\right|^{2} \mathrm{~d} x+\int_{B_{1}}\left|\nabla u_{n}\right|^{2} \mathrm{~d} x+\int_{B_{1}}\left|\Delta u_{n}\right|^{2} \mathrm{~d} x\right)^{\frac{q}{2}} \\
& \leq C_{8} r_{0}^{b_{0}+\frac{4}{\sigma}}\left(\int_{B_{3 / 4} \backslash B_{1 / 2}} V(|x|)\left|u_{n}\right|^{2} \mathrm{~d} x+\int_{B_{1}}\left|\nabla u_{n}\right|^{2} \mathrm{~d} x+\int_{B_{1}}\left|\Delta u_{n}\right|^{2} \mathrm{~d} x\right)^{\frac{q}{2}} \\
& \leq C_{9} r_{0}^{b_{0}+\frac{4}{\sigma}}\left\|u_{n}\right\|^{q},
\end{aligned}
$$


where the fact that $\inf _{B_{3 / 4} \backslash B_{1 / 2}} V>0$ has been used. Next, we shall estimate the integral on $B_{R} \backslash B_{r_{0}}$. By the continuity of the functions $V$ and $K$ and the continuous embedding $H^{2}\left(B_{R} \backslash\right.$ $\left.B_{r_{0}}\right) \hookrightarrow L^{q}\left(B_{R} \backslash B_{r_{0}}\right)$, we infer

$$
\int_{B_{R} \backslash B_{r_{0}}} K(|x|)\left|u_{n}\right|^{q} \mathrm{~d} x \leq C_{10} \int_{B_{R} \backslash B_{r_{0}}}\left|u_{n}\right|^{q} \mathrm{~d} x \leq C_{11}\left\|u_{n}\right\|^{q} .
$$

Combining (2.5), (2.7) and (2.8), we deduce that

$$
\int_{\mathbb{R}^{4}} K(|x|)\left|u_{n}\right|^{q} \mathrm{~d} x \leq C_{12}\left(R^{b-a-(q-2) \frac{a+6}{4}}+r_{0}^{b_{0}+\frac{4}{\sigma}}+1\right)\left\|u_{n}\right\|^{q}=o_{n}(1) .
$$

Hence,

$$
\lim _{n \rightarrow \infty} \int_{\mathbb{R}^{4}} K(|x|)\left|u_{n}\right|^{q} \mathrm{~d} x=0,
$$

which contradicts the fact that $\left|u_{n}\right|_{L^{q}\left(\mathbb{R}^{4} ; K\right)}=1$. Consequently, $S_{q}>0$ and this proves the continuity of the embedding. To prove the compacity, let $\left(u_{n}\right)$ be a bounded sequence in $E$. Thus, there exists $u \in E$ such that, up to subsequence, $u_{n} \rightarrow u$ weakly in $E$. Without loss of generality, we may suppose that $u=0$. As in (2.5), we have

$$
\int_{B_{R}^{c}} K(|x|)\left|u_{n}\right|^{q} \mathrm{~d} x \leq C_{13} R^{b-a-(q-2) \frac{a+6}{4}}\left\|u_{n}\right\|^{q} \leq C_{14} R^{b-a-(q-2) \frac{a+6}{4}} .
$$

Since $b-a-\frac{(q-2)(a+6)}{4}<0$, given $\epsilon>0$, we can take $R>0$ sufficiently large such that

$$
\int_{B_{R}^{c}} K(|x|)\left|u_{n}\right|^{q} \mathrm{~d} x<\frac{\epsilon}{3} .
$$

On the other hand, by (2.7), we have

$$
\int_{B_{r_{0}}} K(|x|)\left|u_{n}\right|^{q} \mathrm{~d} x \leq C_{15} r_{0}^{b_{0}+\frac{4}{\sigma}}\left\|u_{n}\right\|^{q} \leq C_{16} r_{0}^{b_{0}+\frac{4}{\sigma}} .
$$

Thus, for $r_{0}$ chosen sufficiently small, it follows

$$
\int_{B_{r_{0}}} K(|x|)\left|u_{n}\right|^{q} \mathrm{~d} x<\frac{\epsilon}{3} .
$$

Furthermore, by the compactness of the embedding $H^{2}\left(B_{R} \backslash B_{r_{0}}\right)$ into $L^{q}\left(B_{R} \backslash B_{r_{0}}\right)$ together with the fact that $\inf _{B_{R} \backslash B_{r_{0}}} K>0$, there exists $n_{0} \in \mathbb{N}$ such that if $n>n_{0}$, then

$$
\int_{B_{R} \backslash B_{r_{0}}} K(|x|)\left|u_{n}\right|^{q} \mathrm{~d} x<\frac{\epsilon}{3} .
$$

From (2.9), (2.10) and (2.11), we deduce that

$$
\int_{\mathbb{R}^{4}} K(|x|)\left|u_{n}\right|^{q} \mathrm{~d} x<\epsilon, \quad \forall n>n_{0} .
$$

Therefore, $u_{n} \rightarrow 0$ in $L^{q}\left(\mathbb{R}^{4} ; K\right)$ and this finalizes the proof of Lemma 2.3.

Remark 2.4 Besides their important role in order to establish our Adams' inequality, in this remark we give some additional comments concerning the ranges of the parameters $a, a_{0}, b$ and $b_{0}$. 
1. The condition $b_{0}>-4$ is necessary to get the continuity of the embeddings $E \hookrightarrow$ $L^{q}\left(\mathbb{R}^{4} ; K\right), q \geq 2$. In fact, suppose that $b_{0} \leq-4$ and $K(r) \equiv r^{b_{0}}$. Take a radial function $\varphi \in C_{0}^{\infty}\left(\mathbb{R}^{4}\right)$ such that $\inf _{0<r<\epsilon_{0}} \varphi(r)>0$ for some $\epsilon_{0}>0$ sufficiently small. We have

$$
\int_{0}^{+\infty} K(r) \varphi^{2}(r) r^{3} \mathrm{~d} r \geq \int_{0}^{\epsilon_{0}} r^{b_{0}+3} \varphi^{2}(r) \mathrm{d} r=+\infty
$$

Consequently, $E$ is not continuously immersed in $L^{2}\left(\mathbb{R}^{4} ; K\right)$.

2. The condition $a_{0}>-4$ is necessary to obtain that $C_{0, \text { rad }}^{\infty}\left(\mathbb{R}^{4}\right):=\left\{u \in C_{0}^{\infty}\left(\mathbb{R}^{4}\right)\right.$ : $u$ is radial $\} \subset E$ which plays a capital role in our functional setting.

3. The condition $b<a$ is necessary to obtain the compactness of the embeddings $E \hookrightarrow$ $L^{q}\left(\mathbb{R}^{4} ; K\right), q \geq 2$. In fact, suppose that $V(r)=K(r)=1, \forall r \in(0,+\infty)$. Thus, one can choose $a=0$. Clearly, $\limsup _{r \rightarrow+\infty} \frac{K(r)}{r^{b}}<+\infty, \forall b \geq 0$. Therefore, it suffices to note that, in this case, $E=H_{\text {rad }}^{2}\left(\mathbb{R}^{4}\right)$ and to use the fact that the embedding $H_{\text {rad }}^{2}\left(\mathbb{R}^{4}\right) \hookrightarrow$ $L^{2}\left(\mathbb{R}^{4}\right)$ is not compact.

4. Finally, the condition $a>-6$ is purely technical and it is highly needed to get our radial Lemma 2.1 which is widely used in the proof of our main results. This is the best range if we want to control the radial function by some (negative) exponent of the variable's norm in $\mathbb{R}^{4}$. The authors believe that this range is quite good and almost the best accessible one.

\section{Adams' inequality: the Proof of Theorem 1.2}

Let $\alpha \leq \alpha_{0}$. Recall that by the hypothesis $(K)$, we have

$$
K(|x|) \leq C|x|^{b} \text {, for }|x| \geq R_{0} \text {, and } K(|x|) \leq C|x|^{b_{0}} \text {, for } 0<|x| \leq r_{0} .
$$

Let $R>1$ to be chosen later and set

$$
I_{1}(\alpha, u)=\int_{B_{R}} K(|x|)\left(e^{\alpha u^{2}}-1\right) \mathrm{d} x, \quad I_{2}(\alpha, u)=\int_{B_{R}^{c}} K(|x|)\left(e^{\alpha u^{2}}-1\right) \mathrm{d} x .
$$

Thus,

$$
\int_{\mathbb{R}^{4}} K(|x|)\left(e^{\alpha u^{2}}-1\right) \mathrm{d} x=I_{1}(\alpha, u)+I_{2}(\alpha, u) .
$$

Now, we are going to estimate $I_{1}(\alpha, u)$ and $I_{2}(\alpha, u)$. First, using (3.1) and the continuity of the embedding $E \hookrightarrow L^{2}\left(\mathbb{R}^{4} ; K\right)$, for $u \in E$, one has

$$
\begin{aligned}
I_{2}(\alpha, u) & =\int_{B_{R}^{c}} K(|x|) \sum_{j=1}^{\infty} \frac{\alpha^{j} u^{2 j}}{j !} \mathrm{d} x=\int_{B_{R}^{c}} K(|x|) \sum_{j=2}^{\infty} \frac{\alpha^{j} u^{2 j}}{j !} \mathrm{d} x+\alpha \int_{B_{R}^{c}} K(|x|) u^{2} \mathrm{~d} x \\
& \leq C_{0} \int_{B_{R}^{c}}|x|^{b} \sum_{j=2}^{\infty} \frac{\alpha^{j} u^{2 j}}{j !} \mathrm{d} x+C_{1}\|u\|^{2}=C_{0} \sum_{j=2}^{\infty} \frac{\alpha^{j}}{j !} \int_{B_{R}^{c}}|x|^{b} u^{2 j} \mathrm{~d} x+C_{1}\|u\|^{2} .
\end{aligned}
$$


By the virtue of Lemma 2.1 and using the fact that $a>-6$ and $b-j \frac{a+6}{2}+4<b-a<0$, for all $j \geq 2$, we get

$$
\begin{aligned}
\int_{B_{R}^{c}}|x|^{b} u^{2 j} & \leq(C\|u\|)^{2 j} \int_{B_{R}^{c}}|x|^{b-j \frac{a+6}{2}} \mathrm{~d} x=2 \pi^{2}(C\|u\|)^{2 j} \int_{R}^{\infty} s^{b-j \frac{a+6}{2}+3} \mathrm{~d} s \\
& =2 \pi^{2}(C\|u\|)^{2 j} \frac{R^{b-j \frac{a+6}{2}+4}}{j \frac{a+6}{2}-b-4} \leq \frac{2 \pi^{2}}{(a-b) R^{a-b}}(C\|u\|)^{2 j},
\end{aligned}
$$

and then

$$
\begin{aligned}
I_{2}(\alpha, u) & \leq \frac{2 \pi^{2} C_{0}}{(a-b) R^{a-b}} \sum_{j=2}^{\infty} \frac{\left(\alpha C^{2}\|u\|^{2}\right)^{j}}{j !}+C_{1}\|u\|^{2} \\
& =\frac{2 \pi^{2} C_{0}}{(a-b) R^{a-b}}\left(e^{\alpha C^{2}\|u\|^{2}}-1-\alpha C^{2}\|u\|^{2}\right)+C_{1}\|u\|^{2} .
\end{aligned}
$$

Hence, $I_{2}(\alpha, u)<+\infty, \forall u \in E$. Moreover,

$$
\sup _{u \in E,\|u\| \leq 1} I_{2}(\alpha, u)<+\infty, \forall \alpha>0 .
$$

Next, we shall estimate the integral $I_{1}(\alpha, u)$. Here, two cases have to be analyzed:

Case $1 b_{0} \geq 0$. In view of (3.1), there exists a positive constant $C$ depending on $R$ such that

$$
I_{1}(\alpha, u) \leq C \int_{B_{R}} e^{\alpha u^{2}} \mathrm{~d} x .
$$

Let $v(x)=u(x)-u(R), x \in B_{R}$. For $x \in B_{R}$, we have

$u^{2}(x)=(v(x)+u(R))^{2}=v^{2}(x)+u^{2}(R)+2 v(x) u(R) \leq\left(1+u^{2}(R)\right) v^{2}(x)+u^{2}(R)+1$.

Using Remark 2.2, then for $R>R_{0}$, we get

$$
u^{2}(x) \leq\left(1+C R^{-\frac{a+6}{2}} \mathcal{V}^{2}(u)\right) v^{2}(x)+C R^{-\frac{a+6}{2}}\|u\|^{2}+1, \quad \forall x \in B_{R} .
$$

Choosing $R$ large enough such that $C R^{-\frac{a+6}{2}} \leq 1$, we infer

$$
u^{2}(x) \leq\left(1+C R^{-\frac{a+6}{2}} \mathcal{V}^{2}(u)\right) v^{2}(x)+\|u\|^{2}+1, \quad \forall x \in B_{R} .
$$

Putting that last inequality in (3.3), we obtain

$$
I_{1}(\alpha, u) \leq C_{1} e^{\alpha\|u\|^{2}} \int_{B_{R}} e^{\alpha\left(1+C R^{-\frac{a+6}{2}} \mathcal{V}^{2}(u)\right) v^{2}(x)} \mathrm{d} x .
$$

On the other hand, it is easy to see that $v \in H^{2}\left(B_{R}\right) \cap H_{0}^{1}\left(B_{R}\right)$. Hence, we can apply the classical Adams' inequality (see (1.8)) in (3.4) to deduce that

$$
I_{1}(\alpha, u)<+\infty, \forall u \in E .
$$

Now, let $u \in E$ be such that $\|u\|^{2}=|\Delta u|_{2}^{2}+\mathcal{V}^{2}(u) \leq 1$. Set $w(x)=\sqrt{1+C R^{-\frac{a+6}{2}} \mathcal{V}^{2}(u)}$ $v(x), x \in B_{R}$. By construction, we have

$$
\int_{B_{R}}|\Delta v|^{2} \mathrm{~d} x=\int_{B_{R}}|\Delta u|^{2} \mathrm{~d} x \leq 1-\mathcal{V}^{2}(u) .
$$


Hence,

$\int_{B_{R}}|\Delta w|^{2} \mathrm{~d} x=\left(1+C R^{-\frac{a+6}{2}} \mathcal{V}^{2}(u)\right) \int_{B_{R}}|\Delta v|^{2} \mathrm{~d} x \leq\left(1+C R^{-\frac{a+6}{2}} \mathcal{V}^{2}(u)\right)\left(1-\mathcal{V}^{2}(u)\right)$.

Choosing $R$ sufficiently large such that $C R^{-\frac{a+6}{2}} \leq 1$, it follows

$$
\int_{B_{R}}|\Delta w|^{2} \mathrm{~d} x \leq\left(1+\mathcal{V}^{2}(u)\right)\left(1-\mathcal{V}^{2}(u)\right) \leq 1 .
$$

Having in mind that $w \in H^{2}\left(B_{R}\right) \cap H_{0}^{1}\left(B_{R}\right)$, we infer from (1.8) that

$$
\int_{B_{R}} e^{\alpha w^{2}} \mathrm{~d} x \leq C\left|B_{R}\right|, \quad \forall \alpha \leq 32 \pi^{2},
$$

where $\left|B_{R}\right|=\int_{B_{R}} \mathrm{~d} x$. That inequality together with (3.4) implies that

$$
\sup _{u \in E,\|u\| \leq 1} I_{1}(\alpha, u)<+\infty, \quad \forall \alpha \leq 32 \pi^{2} .
$$

Case $2-4<b_{0}<0$. Since $0<r_{0}<R_{0}<R$, by (3.1) we obtain

$$
\begin{aligned}
I_{1}(\alpha, u) & =\int_{B_{r_{0}}} K(|x|)\left(e^{\alpha u^{2}}-1\right) \mathrm{d} x+\int_{B_{R} \backslash B_{r_{0}}} K(|x|)\left(e^{\alpha u^{2}}-1\right) \mathrm{d} x \\
& \leq C_{0} \int_{B_{r_{0}}}|x|^{b_{0}} e^{\alpha u^{2}} \mathrm{~d} x+C \int_{B_{R} \backslash B_{r_{0}}} e^{\alpha u^{2}} \mathrm{~d} x \\
& \leq C_{0} \int_{B_{R}}|x|^{b_{0}} e^{\alpha u^{2}} \mathrm{~d} x+C \int_{B_{R}} e^{\alpha u^{2}} \mathrm{~d} x .
\end{aligned}
$$

From the first case, we have

$$
\sup _{u \in E,\|u\| \leq 1} \int_{B_{R}} e^{\alpha u^{2}} \mathrm{~d} x<+\infty, \forall \alpha \leq 8 \pi^{2}\left(4+b_{0}\right) \leq 32 \pi^{2} .
$$

On the other hand, a similar inequality to (3.4) can be immediately established, that is

$$
\int_{B_{R}}|x|^{b_{0}} e^{\alpha u^{2}} \mathrm{~d} x \leq C_{2} e^{\alpha\|u\|^{2}} \int_{B_{R}} \frac{e^{\alpha w^{2}(x)}}{|x|^{-b_{0}}} \mathrm{~d} x .
$$

Now, using again the fact that $w \in H^{2}\left(B_{R}\right) \cap H_{0}^{1}\left(B_{R}\right)$ and that $|\Delta w|_{L^{2}\left(B_{R}\right)} \leq 1$, one can apply the singular Adams' inequality for bounded domains (1.9) to deduce that

$$
\int_{B_{R}} \frac{e^{\alpha w^{2}(x)}}{|x|^{-b_{0}}} \mathrm{~d} x \leq \sup _{u \in W_{N}^{2,2}\left(B_{R}\right),|\Delta u|_{L^{2}\left(B_{R}\right)} \leq 1} \int_{B_{R}} \frac{e^{\alpha u^{2}(x)}}{|x|^{-b_{0}}} \mathrm{~d} x<+\infty, \quad \forall \alpha \leq 8 \pi^{2}\left(4+b_{0}\right),
$$

where $W_{N}^{2,2}\left(B_{R}\right)=H^{2}\left(B_{R}\right) \cap H_{0}^{1}\left(B_{R}\right)$. Combining (3.8), (3.7), (3.6) and (3.5), we obtain

$$
\sup _{u \in E,\|u\| \leq 1} I_{1}(\alpha, u)<+\infty, \quad \forall \alpha \leq 8 \pi^{2}\left(4+b_{0}\right) .
$$

This ends the proof of the first part of Theorem 1.2. Now, we arrive to the sharpness of inequality (1.10). Assume that $-4<b_{0} \leq 0, \liminf _{r \rightarrow 0^{+}} \frac{K(r)}{r^{b_{0}}}>0$. We claim that

$$
\sup _{u \in E,\|u\| \leq 1} \int_{\mathbb{R}^{4}} K(|x|)\left(e^{\alpha u^{2}}-1\right) \mathrm{d} x=+\infty, \quad \forall \alpha>\alpha_{0}=8 \pi^{2}\left(4+b_{0}\right) .
$$


We will consider a family of test functions that has been introduced and used in [23]. For $0<\epsilon<1$, we define

$$
u_{\epsilon}(x)= \begin{cases}\sqrt{\frac{-\log \epsilon}{32 \pi^{2}}}-\frac{|x|^{2}}{\sqrt{-8 \pi^{2} \epsilon \log \epsilon}}+\frac{1}{\sqrt{-8 \pi^{2} \log \epsilon}}, & \text { if }|x|<\epsilon^{1 / 4}, \\ \frac{-\log |x|}{\sqrt{-2 \pi^{2} \log \epsilon}}, & \text { if } \epsilon^{1 / 4} \leq|x| \leq 1, \\ \eta_{\epsilon}, & \text { if }|x|>1,\end{cases}
$$

where $\eta_{\epsilon} \in C_{0}^{\infty}\left(B_{2}\right)$ is such that

$$
\eta_{\epsilon}(x)=0, \quad \frac{\partial \eta_{\epsilon}}{\partial v}(x)=\frac{1}{\sqrt{-2 \pi^{2} \log \epsilon}}, \quad \forall x \in \mathbb{R}^{4}, \quad|x|=1,
$$

and $\left|\eta_{\epsilon}\right|_{\infty},\left|\nabla \eta_{\epsilon}\right|_{\infty}$ and $\left|\Delta \eta_{\epsilon}\right|_{\infty}$ are all $O\left(\frac{1}{\sqrt{-\log \epsilon}}\right)$ as $\epsilon \rightarrow 0^{+}$. Clearly, $u_{\epsilon} \in D_{\text {rad }}^{1,2}\left(\mathbb{R}^{4}\right)$ and $\int_{\mathbb{R}^{4}}\left|\Delta u_{\epsilon}\right|^{2} \mathrm{~d} x<+\infty$. An easy computation gives

$$
\left|\nabla u_{\epsilon}\right|_{2}^{2}=O\left(\frac{-1}{\log \epsilon}\right), \quad\left|\Delta u_{\epsilon}\right|_{2}^{2}=1+O\left(\frac{-1}{\log \epsilon}\right) .
$$

On the other hand since $\limsup _{r \rightarrow 0^{+}} \frac{V(r)}{r^{a_{0}}}<+\infty$, then, without loss of generality, one has

$$
V(|x|) \leq C_{0}|x|^{a_{0}}, \quad \forall 0<|x|<r_{0} .
$$

Recall that $r_{0}$ has been chosen such that $r_{0}<\frac{1}{2}$. Thus,

$$
\begin{aligned}
& \int_{\mathbb{R}^{4}} V(|x|) u_{\epsilon}^{2}(x) \mathrm{d} x \leq C_{1}(-\log \epsilon) \int_{0}^{\epsilon^{1 / 4}} s^{a_{0}+3} \mathrm{~d} s+\left(\frac{-C_{1}}{\log \epsilon}\right) \int_{0}^{\epsilon^{1 / 4}} s^{a_{0}+3}\left(1-\frac{s^{2}}{\sqrt{\epsilon}}\right)^{2} \mathrm{~d} s \\
& +\left(\frac{-C_{1}}{\log \epsilon}\right) \int_{\epsilon^{1 / 4}}^{1} s^{a_{0}+3}|\log s| \mathrm{d} s+C_{1}\left|\eta_{\epsilon}\right|_{\infty}^{2} \int_{1}^{2} s^{a_{0}+3} \mathrm{~d} s .
\end{aligned}
$$

Since $a_{0}>-4$, (3.11) implies that $u_{\epsilon} \in E$. Taking again into account that $a_{0}>-4$, we can easily see that $(\log \epsilon) \epsilon^{\frac{a_{0}+4}{4}} \rightarrow 0$, as $\epsilon \rightarrow 0^{+}$. On the other hand, one has

$$
\left(\frac{-C_{1}}{\log \epsilon}\right) \int_{0}^{\epsilon^{1 / 4}} s^{a_{0}+3}\left(1-\frac{s^{2}}{\sqrt{\epsilon}}\right)^{2} \mathrm{~d} s \leq\left(\frac{-C_{1}}{\log \epsilon}\right) \int_{0}^{\epsilon^{1 / 4}} s^{a_{0}+3} \mathrm{~d} s \rightarrow 0, \text { as } \epsilon \rightarrow 0^{+} .
$$

Also, the fact that $a_{0}>-4$ implies that the function $s \longmapsto s^{a_{0}+3}|\log s|$ is integrable on ]0, 1[. Consequently,

$$
\left(\frac{-C_{1}}{\log \epsilon}\right) \int_{\epsilon^{1 / 4}}^{1} s^{a_{0}+3}|\log s| \mathrm{d} s \rightarrow 0, \quad \text { as } \epsilon \rightarrow 0^{+} .
$$

Finally, noting that $\left|\eta_{\epsilon}\right|_{\infty} \rightarrow 0$, as $\epsilon \rightarrow 0^{+}$, we deduce from (3.11) that

$$
\int_{\mathbb{R}^{4}} V(|x|) u_{\epsilon}^{2}(x) \mathrm{d} x \rightarrow 0, \quad \text { as } \epsilon \rightarrow 0^{+} .
$$

Combining (3.10) and (3.12), it follows

$$
\left\|u_{\epsilon}\right\|^{2} \rightarrow 1, \text { as } \epsilon \rightarrow 0^{+} .
$$


Next, by the condition added concerning the behavior of $K$ near 0 , there exists a positive constant $C_{2}$ such that

$$
K(|x|) \geq C_{2}|x|^{b_{0}}, \quad \forall 0<|x|<r_{0} .
$$

Set $\tilde{u_{\epsilon}}=\frac{u_{\epsilon}}{\left\|u_{\epsilon}\right\|}$. Observing that $\tilde{u_{\epsilon}}(x) \geq \frac{\sqrt{\frac{-\log \epsilon}{32 \pi^{2}}}}{\left\|u_{\epsilon}\right\|}, \forall x \in \mathbb{R}^{4},|x|<\epsilon^{1 / 4}$, we infer

$$
\begin{aligned}
\int_{\mathbb{R}^{4}} K(|x|)\left(e^{\alpha \tilde{u_{\epsilon}}}-1\right) \mathrm{d} x & \geq C_{2} \int_{|x|<\epsilon}|x|^{b_{0}}\left(e^{\frac{-\alpha \log \epsilon}{32 \pi^{2}\left\|u_{\epsilon}\right\|^{2}}}-1\right) \mathrm{d} x \\
& =C_{2}\left(e^{\frac{-\alpha \log \epsilon}{32 \pi^{2}\left\|u_{\epsilon}\right\|^{2}}}-1\right) \epsilon^{\frac{b_{0}+4}{4}} \\
& =C_{2} \epsilon^{\frac{b_{0}+4}{4}-\frac{\alpha}{32 \pi^{2}\left\|u_{\epsilon}\right\|^{2}}}-C_{2} \epsilon^{\frac{b_{0}+4}{4}} .
\end{aligned}
$$

Since $b_{0}+4>0$ and $\left\|u_{\epsilon}\right\|^{2} \rightarrow 1$, as $\epsilon \rightarrow 0^{+}$, from (3.13) we deduce that

$$
\lim _{\epsilon \rightarrow 0^{+}} \int_{\mathbb{R}^{4}} K(|x|)\left(e^{\alpha \tilde{u}^{2}}-1\right) \mathrm{d} x=+\infty, \quad \forall \alpha>8 \pi^{2}\left(b_{0}+4\right) .
$$

Taking into account that

$$
\sup _{u \in E,\|u\| \leq 1} \int_{\mathbb{R}^{4}} K(|x|)\left(e^{\alpha u^{2}}-1\right) \mathrm{d} x \geq \int_{\mathbb{R}^{4}} K(|x|)\left(e^{\alpha \tilde{u}_{\epsilon}^{2}}-1\right) \mathrm{d} x, \quad \forall 0<\epsilon<1,
$$

then our sharpness result can immediately be derived from (3.14). A consequence of Theorem 1.2 , which will be very useful in our application, is given by the following corollary:

Corollary 3.1 Under the assumptions of Theorem 1.2, if $u \in E$ is such that $\|u\| \leq\left(\frac{\alpha_{0}}{\alpha}\right)^{\frac{1}{2}}$, then there exists a constant $C=C(\alpha)>0$ independent of $u$ such that

$$
\int_{\mathbb{R}^{4}} K(|x|)\left(e^{\alpha u^{2}}-1\right) \mathrm{d} x \leq C .
$$

\section{An application to a quasilinear elliptic problem}

In this section, we will apply Theorem 1.2 and Lemma 2.3 to study the following elliptic semilinear nonhomogeneous equation

$$
\Delta^{2} u-\Delta u+V(|x|) u=K(|x|) f(u)+V(|x|) h(|x|), \quad x \in \mathbb{R}^{4},
$$

where $\left(F_{1}\right) \lim _{s \rightarrow 0} \frac{f(s)}{s}=0$.

$\left(F_{2}\right)$ There exist $C_{0}>0, \beta>0$ and $s_{0}>0$ such that

$$
|f(s)| \leq C_{0} e^{\beta s^{2}}, \forall|s| \geq s_{0} .
$$

$\left(H_{1}\right) h \in L_{\text {rad }}^{2}\left(\mathbb{R}^{4} ; V\right)$.

Nowadays, the importance of the study of higher-order elliptic equations and especially those involving the biharmonic operator (i.e., of fourth order) is well confirmed. In reality, such equations arise in applications from conformal geometry and mathematical physics because they can be used to describe the mechanical vibrations of an elastic plate, the traveling waves in a suspension bridge or the static deflection of an elastic plate in a fluid. For this aspect of the polyharmonic equations, the reader is invited to see $[11,15,16,21]$ and references 
therein. Many works have dealt with elliptic equations similar to (4.1) but with some strong coercivity conditions on the potential $V$ in order to recover the compactness lost by the unboundedness of the domain. We refer, for example, to [27,38,39]. Very recently, the case when the nonlinearity enjoys an exponential growth at infinity has known a big interest. We can cite $[7-9,18,20,30,31,37]$. But, to the very best of our knowledge, it seems that there is no work dealing with the existence of solutions for nonhomogeneous biharmonic equations involving simultaneously an exponential nonlinearity and vanishing potentials. In fact, this case needs a new Adams' inequality appropriate to this new situation. In this section, profiting of the Adams' inequality established in Theorem 1.2, we prove two existence results for the equation (4.1).

A weak solution of Eq. (4.1) is a function $u \in E$ such that

$$
\int_{\mathbb{R}^{4}}(\Delta u \Delta v+\nabla u \cdot \nabla v+V(|x|) u v) \mathrm{d} x=\int_{\mathbb{R}^{4}}(K(|x|) f(u)+V(|x|) h(|x|)) v \mathrm{~d} x,
$$

for all $v \in E$. Clearly, the weak solutions of (4.1) correspond to the critical points of the $C^{1}$ energy functional $I: E \rightarrow \mathbb{R}$ defined by

$$
I(u)=\frac{\|u\|^{2}}{2}-\int_{\mathbb{R}^{4}}(K(|x|) F(u)+V(|x|) h(|x|) u) \mathrm{d} x,
$$

where $F$ stands for the primitive of $f$ vanishing at 0 , that is, $F(s)=\int_{0}^{s} f(t) \mathrm{d} t, s \in \mathbb{R}$. Here, we state our existence results.

Proposition 4.1 Assume that $(V)-(K),\left(F_{1}\right)-\left(F_{2}\right)$ and $\left(H_{1}\right)$ hold. Then, there exists $m>0$ such that Eq. (4.1) has at least one nontrivial solution $u$ such that $I(u)<0$ provided that $0<|h|_{L^{2}\left(\mathbb{R}^{4} ; V\right)}<m$.

In the second existence result, we prove that if we add two hypotheses, then Eq. (4.1) has a weak solution of positive energy. The first one:

$\left(F_{3}\right)$ There exist $\mu>0$ and $p>2$ such that

$$
F(s) \geq \mu|s|^{p}, \forall s \in \mathbb{R},
$$

has been introduced by Cao [10] and it prescribes the growth of $f$ near the origin. The second one is the well-known Ambrosetti-Rabinowitz (AR) condition:

$\left(F_{4}\right)$ There exists $\theta>2$ such that

$$
\theta F(s) \leq f(s) s, \forall s \in \mathbb{R} .
$$

In this case, we have the following existence result.

Proposition 4.2 In addition to the hypotheses in Proposition 4.1, assume that $\left(F_{3}\right)$ and $\left(F_{4}\right)$ hold. Then, there exists $\mu_{0}>0$ such that, if $0 \leq|h|_{L^{2}\left(\mathbb{R}^{4} ; V\right)}<m$ and $\mu>\mu_{0}$, then Eq. (4.1) has a weak solution of positive energy.

\subsection{Weak solution of negative energy}

In this part, we prove Proposition 4.1. As a first step in the proof, we claim that there exist $\gamma>0, \rho>0$ and $m>0$ such that, if $|h|_{L^{2}\left(\mathbb{R}^{4} ; V\right)}<m$, then

$$
I(u) \geq \gamma, \quad \forall u \in E, \quad\|u\|=\rho .
$$


By $\left(F_{1}\right)$ and $\left(F_{2}\right)$, for all $\epsilon>0$, one can find $c_{\epsilon}>0$ such that

$$
|F(s)| \leq \epsilon s^{2}+c_{\epsilon} s^{3}\left(e^{\beta s^{2}}-1\right), \quad \forall s \in \mathbb{R} .
$$

Let $r_{1}>1$ and $r_{2}>1$ be such that $\frac{1}{r_{1}}+\frac{1}{r_{2}}=1$. By Hölder's inequality and (4.3), for all $u \in E$, we infer

$$
\begin{aligned}
\int_{\mathbb{R}^{4}} K(|x|) F(u) \mathrm{d} x \leq & \epsilon \int_{\mathbb{R}^{4}} K(|x|) u^{2} \mathrm{~d} x \\
& +c_{\epsilon}^{\prime}\left(\int_{\mathbb{R}^{4}} K(|x|)|u|^{3 r_{1}} \mathrm{~d} x\right)^{\frac{1}{r_{1}}}\left(\int_{\mathbb{R}^{4}} K(|x|)\left(e^{\beta r_{2} u^{2}}-1\right) \mathrm{d} x\right)^{\frac{1}{r_{2}}} .
\end{aligned}
$$

The continuity of the embeddings $E \hookrightarrow L^{2}\left(\mathbb{R}^{4} ; K\right)$ and $E \hookrightarrow L^{3 r_{1}}\left(\mathbb{R}^{4} ; K\right)$ together with Corollary 3.1 implies

$$
\int_{\mathbb{R}^{4}} K(|x|) F(u) \mathrm{d} x \leq C_{1} \epsilon\|u\|^{2}+C_{2}(\epsilon)\|u\|^{3}, \quad \forall u \in E, \quad\|u\| \leq\left(\frac{\alpha_{0}}{\beta r_{2}}\right)^{\frac{1}{2}} .
$$

By (4.4), one has

$$
I(u) \geq\left(\frac{1}{2}-C_{1} \epsilon\right)\|u\|^{2}-C_{2}(\epsilon)\|u\|^{3}-|h|_{L^{2}\left(\mathbb{R}^{4} ; V\right)}\|u\|, \quad \forall u \in E, \quad\|u\| \leq\left(\frac{\alpha_{0}}{\beta r_{2}}\right)^{\frac{1}{2}} .
$$

Choosing $\epsilon=\frac{1}{4 C_{1}}$, we obtain

$$
I(u) \geq \frac{1}{4}\|u\|^{2}-C_{3}\|u\|^{3}-|h|_{L^{2}\left(\mathbb{R}^{4} ; V\right)}\|u\|, \quad \forall u \in E, \quad\|u\| \leq\left(\frac{\alpha_{0}}{\beta r_{2}}\right)^{\frac{1}{2}} .
$$

Taking $0<\rho<\min \left(\frac{1}{8 C_{3}},\left(\frac{\alpha_{0}}{\beta r_{2}}\right)^{\frac{1}{2}}\right)$, it follows

$$
I(u) \geq \rho\left(\frac{\rho}{8}-|h|_{L^{2}\left(\mathbb{R}^{4} ; V\right)}\right), \quad \forall u \in E, \quad\|u\|=\rho .
$$

Consequently, one can take $0<m<\frac{\rho}{8}$ and (4.2) follows. Now, by (4.2), for $|h|_{L^{2}\left(\mathbb{R}^{4}, V\right)}<m$, we have

$$
\inf _{u \in E,\|u\|=\rho} I(u) \geq \gamma>0=I(0) \geq \inf _{u \in E,\|u\|<\rho} I(u) .
$$

Thus, one can apply the Ekeland's variational principle (see [14]) to assert the existence of a (PS) sequence $\left(u_{n}\right) \subset\{v \in E,\|v\| \leq \rho\}$ of $I$ at the level

$$
d=\inf _{u \in E,\|u\| \leq \rho} I(u),
$$

that is,

$$
I\left(u_{n}\right) \rightarrow d, \quad I^{\prime}\left(u_{n}\right) \rightarrow 0, \quad \text { as } n \rightarrow+\infty .
$$

By $\left(F_{1}\right)$ and $\left(F_{2}\right)$, one has

$$
\begin{aligned}
& \int_{\mathbb{R}^{4}} K(|x|)\left(f\left(u_{n}\right)\right)^{2} \mathrm{~d} x \leq C_{4} \int_{\mathbb{R}^{4}} K(|x|)\left(u_{n}^{2}+u_{n}^{2}\left(e^{2 \beta u_{n}^{2}}-1\right)\right) \mathrm{d} x \\
& \quad \leq C_{4} \int_{\mathbb{R}^{4}} K(|x|) u_{n}^{2} \mathrm{~d} x+C_{5}\left(\int_{\mathbb{R}^{4}} K(|x|) u_{n}^{4} \mathrm{~d} x\right)^{\frac{1}{2}}\left(\int_{\mathbb{R}^{4}} K(|x|)\left(e^{4 \beta u_{n}^{2}}-1\right)\right)^{\frac{1}{2}} \mathrm{~d} x .
\end{aligned}
$$


Plainly, one could choose $\rho$ small enough such that $\rho \leq\left(\frac{\alpha_{0}}{4 \beta}\right)^{\frac{1}{2}}$. Hence, in view of Corollary 3.1 and taking the continuity of the embeddings $E \hookrightarrow L^{2}\left(\mathbb{R}^{4} ; K\right)$ and $E \hookrightarrow L^{4}\left(\mathbb{R}^{4} ; K\right)$ into account, we deduce from (4.5) that

$$
\sup _{n \in \mathbb{N}} \int_{\mathbb{R}^{4}} K(|x|)\left(f\left(u_{n}\right)\right)^{2} \mathrm{~d} x<+\infty .
$$

Since $\left(u_{n}\right)$ is bounded in $E$, then, up to a subsequence, there exists $u \in E$ such that $u_{n} \rightarrow u$ weakly in $E$. Using (4.6) together with Hölder's inequality, we infer

$$
\left|\int_{\mathbb{R}^{4}} K(|x|) f\left(u_{n}\right)\left(u_{n}-u\right) \mathrm{d} x\right| \leq\left(\sup _{n \in \mathbb{N}} \int_{\mathbb{R}^{4}} K(|x|)\left(f\left(u_{n}\right)\right)^{2} \mathrm{~d} x\right)^{\frac{1}{2}}\left|u_{n}-u\right|_{L^{2}\left(\mathbb{R}^{4} ; K\right)} .
$$

Now, the compactness of the embedding $E \hookrightarrow L^{2}\left(\mathbb{R}^{4} ; K\right)$ established in Lemma 2.3 implies

$$
\lim _{n \rightarrow+\infty} \int_{\mathbb{R}^{4}} K(|x|) f\left(u_{n}\right)\left(u_{n}-u\right) \mathrm{d} x=0 .
$$

Since $\left\langle I^{\prime}\left(u_{n}\right), u_{n}-u\right\rangle \rightarrow 0$, as $n \rightarrow+\infty$, then in view of (4.7) it follows

$$
\int_{\mathbb{R}^{4}}\left(\Delta u_{n} \Delta\left(u_{n}-u\right)+\nabla u_{n} \cdot \nabla\left(u_{n}-u\right)+V(|x|) u_{n}\left(u_{n}-u\right)\right) \mathrm{d} x \rightarrow 0, n \rightarrow+\infty .
$$

On the other hand, the weak convergence of $\left(u_{n}\right)$ to $u$ in $E$ leads to

$$
\int_{\mathbb{R}^{4}}\left(\Delta u \Delta\left(u_{n}-u\right)+\nabla u \cdot \nabla\left(u_{n}-u\right)+V(|x|) u\left(u_{n}-u\right)\right) \mathrm{d} x \rightarrow 0, n \rightarrow+\infty .
$$

Therefore,

$$
\left\|u_{n}-u\right\|^{2} \rightarrow 0, n \rightarrow+\infty .
$$

By the continuity of $I$ and $I^{\prime}$, we deduce that $I(u)=d$ and $I^{\prime}(u)=0$. It remains to show that $d<0$. Consider a function $\varphi_{0} \in E$ such that $\varphi_{0} \neq 0$ and $\int_{\mathbb{R}^{4}} V(|x|) h(|x|) \varphi_{0} \mathrm{~d} x>0$. Observe that by (4.4), one has

$$
\frac{1}{t} \int_{\mathbb{R}^{4}} K(|x|) F\left(t \varphi_{0}\right) \mathrm{d} x \rightarrow 0, t \rightarrow 0^{+} .
$$

Hence,

$$
\frac{I\left(t \varphi_{0}\right)}{t} \rightarrow-\int_{\mathbb{R}^{4}} V(|x|) h(|x|) \varphi_{0} \mathrm{~d} x<0, t \rightarrow 0^{+} .
$$

Therefore, $I\left(t \varphi_{0}\right)<0$ for $t$ sufficiently small. This ends the proof of Proposition 4.1.

\subsection{Weak solution of positive energy}

In this subsection, we prove Proposition 4.2. For that aim, let $\varphi_{0}$ be the nontrivial function in $E$ used in the proof of Proposition 4.1. By $\left(F_{3}\right)$, we have

$$
I\left(t \varphi_{0}\right) \leq t^{2} \frac{\left\|\varphi_{0}\right\|^{2}}{2}-t \int_{\mathbb{R}^{4}} V(|x|) h(|x|) \varphi_{0} \mathrm{~d} x-\mu t^{p} \int_{\mathbb{R}^{4}} K(|x|)\left|\varphi_{0}\right|^{p} \mathrm{~d} x, \forall t \geq 0 .
$$

Since $p>2$, then one can find $t_{1}>0$ sufficiently large such that $t_{1}>\frac{\rho}{\left\|\varphi_{0}\right\|}$ and $I\left(t_{1} \varphi_{0}\right)<0$, where $\rho$ is given by (4.2). Hence, by the mountain pass theorem without the Palais-Smale 
condition (see [5]), there exists a sequence $\left(u_{n}\right) \subset E$ such that $I^{\prime}\left(u_{n}\right) \rightarrow 0$ and $I\left(u_{n}\right) \rightarrow c$, as $n \rightarrow+\infty$, with

$$
c=\inf _{g \in \Gamma} \max _{0 \leq t \leq 1} I(g(t)), \Gamma=\left\{g \in C([0,1], E), g(0)=0, g(1)=t_{1} \varphi_{0}\right\} .
$$

Clearly, $c \geq \gamma$. We claim that $c=c(\mu) \rightarrow 0$ as $\mu \rightarrow+\infty$. By the definition of $c$, we have

$$
c \leq \max _{t \geq 0}\left(\frac{\left\|\varphi_{0}\right\|^{2}}{2} t^{2}-\left(\mu \int_{\mathbb{R}^{4}} K(|x|)\left|\varphi_{0}\right|^{p} \mathrm{~d} x\right) t^{p}\right)=\left(\frac{2 A_{1}}{p A_{2}}\right)^{\frac{2}{p-2}} A_{1}\left(1-\frac{2}{p}\right),
$$

where $A_{1}=\frac{\left\|\varphi_{0}\right\|^{2}}{2}, A_{2}=\mu \int_{\mathbb{R}^{4}} K(|x|)\left|\varphi_{0}\right|^{p} \mathrm{~d} x$. Our claim follows immediately. In the presence of the well-known Ambrosetti-Rabinowitz (AR) (condition $\left(F_{4}\right)$ ), the boundedness of the (PS) sequence $\left(u_{n}\right)$ is evident. In fact, it suffices, as usual, to estimate the sequence

$$
I\left(u_{n}\right)-\frac{1}{\theta}\left\langle I^{\prime}\left(u_{n}\right), u_{n}\right\rangle \text {. }
$$

By Young's inequality, there exists a positive constant $C_{1}$ such that

$$
\int_{\mathbb{R}^{4}} V(|x|)|h(|x|)|\left|u_{n}\right| \mathrm{d} x \leq \frac{1}{2}\left(\frac{1}{2}-\frac{1}{\theta}\right)\left\|u_{n}\right\|^{2}+C_{1}|h|_{L^{2}\left(\mathbb{R}^{4} ; V\right)}^{2}, \quad \forall n \in \mathbb{N} .
$$

By the boundedness of the sequence $\left(u_{n}\right)$ in $E$, it follows

$$
\begin{aligned}
I\left(u_{n}\right) & =I\left(u_{n}\right)-\frac{1}{\theta}\left\langle I^{\prime}\left(u_{n}\right), u_{n}\right\rangle+o_{n}(1) \\
& =\left(\frac{1}{2}-\frac{1}{\theta}\right)\left\|u_{n}\right\|^{2}-\left(1-\frac{1}{\theta}\right) \int_{\mathbb{R}^{4}} V(|x|) h(|x|) u_{n} \mathrm{~d} x+o_{n}(1) .
\end{aligned}
$$

Hence,

$$
\left(\frac{1}{2}-\frac{1}{\theta}\right)\left\|u_{n}\right\|^{2} \leq I\left(u_{n}\right)+\int_{\mathbb{R}^{4}} V(|x|)|h(|x|)|\left|u_{n}\right| \mathrm{d} x+o_{n}(1) .
$$

Putting (4.8) in (4.9), we infer

$$
\frac{1}{2}\left(\frac{1}{2}-\frac{1}{\theta}\right)\left\|u_{n}\right\|^{2} \leq I\left(u_{n}\right)+C_{1}|h|_{L^{2}\left(\mathbb{R}^{4} ; V\right)}^{2}+o_{n}(1) .
$$

Having in mind that $I\left(u_{n}\right) \rightarrow c$, then after passing to the limit in the last inequality, we obtain

$$
\limsup _{n \rightarrow+\infty}\left\|u_{n}\right\| \leq\left(\frac{2 c}{\frac{1}{2}-\frac{1}{\theta}}\right)^{\frac{1}{2}}+\left(\frac{2 C_{1}}{\frac{1}{2}-\frac{1}{\theta}}\right)^{\frac{1}{2}}|h|_{L^{2}\left(\mathbb{R}^{4} ; V\right)} .
$$

Since $c \rightarrow 0$ as $\mu \rightarrow+\infty$, then one can find $\mu_{0}>0$ large enough such that

$$
\left(\frac{2 c}{\frac{1}{2}-\frac{1}{\theta}}\right)^{\frac{1}{2}}<\frac{1}{2}\left(\frac{\alpha_{0}}{4 \beta}\right)^{\frac{1}{2}}, \quad \forall \mu>\mu_{0}
$$

On the other hand, it is clear that one could choose $m>0$ small enough such that

$$
\left(\frac{2 C_{1}}{\frac{1}{2}-\frac{1}{\theta}}\right)^{\frac{1}{2}} m<\frac{1}{2}\left(\frac{\alpha_{0}}{4 \beta}\right)^{\frac{1}{2}}, \quad \forall \mu>\mu_{0} .
$$


Consequently, for $\mu>\mu_{0}$ and $|h|_{L^{2}\left(\mathbb{R}^{4} ; V\right)}<m$, by (4.12), (4.11) and (4.10), we get

$$
\limsup _{n \rightarrow+\infty}\left\|u_{n}\right\|<\left(\frac{\alpha_{0}}{4 \beta}\right)^{\frac{1}{2}} .
$$

In view of (4.5), we see that (4.13) implies that (4.6) and (4.7) hold true. Proceeding exactly as in the proof of Proposition 4.1, we immediately deduce that, up to subsequence, $\left(u_{n}\right)$ is strongly convergent to some point $u \in E$. Therefore, $I^{\prime}(u)=0$ and $I(u)=c \geq \gamma>0$. This ends the proof of Proposition 4.2.

Acknowledgements The authors are very grateful to the anonymous referee for his(her) careful reading of the manuscript and his(her) insightful and constructive remarks and comments that helped to clarify the content and improve the presentation of the results in this paper. The second author is supported by Programa de Incentivo à Pós-Graduacão e Pesquisa(PROPESQ) Edital 2015, UEPB.

\section{References}

1. Adachi, S., Tanaka, K.: Trudinger type inequalities in $\mathbb{R}^{N}$ and their best exponents. Proc. Am. Math. Soc. 128, 2051-2057 (2000)

2. Adams, D.R.: A sharp inequality of J. Moser for higher order derivatives. Ann. Math. (2) 128(2), 385-398 (1988)

3. Albuquerque, F.S.B., Severo, U.B.: Stationary Schrödinger equations in $\mathbb{R}^{2}$ with unbounded or vanishing potentials and involving concave nonlinearities. Complex Var. Elliptic Equ. (Online) 63, 368-390 (2018)

4. Albuquerque, F.S.B., Alves, C.O., Medeiros, E.S.: Nonlinear Schrödinger equation with unbounded or decaying radial potentials involving exponential critical growth in $\mathbb{R}^{2}$. J. Math. Anal. Appl. 409, 10211031 (2014)

5. Ambrosetti, A., Rabinowitz, P.H.: Dual variational methods in critical point theory and applications. J. Funct. Anal. 14, 349-381 (1973)

6. Ambrosetti, A., Felli, V., Malchiodi, A.: Ground states of nonlinear Schrödinger equations with potentials vanishing at infinity. J. Eur. Math. Soc. 7, 117-144 (2005)

7. Aouaoui, S.: On some semilinear equation in $\mathbb{R}^{4}$ containing a Laplacian term and involving nonlinearity with exponential growth. Commun. Pure Appl. Anal. 14, 2185-2201 (2015)

8. Aouaoui, S.: Multiplicity result to some Kirchhoff-type biharmonic equation involving exponential growth conditions. Bull. Iran. Math. Soc. 42, 1559-1569 (2016)

9. Aouaoui, S.: A multiplicity result for some integro-differential biharmonic equation in $\mathbb{R}^{4}$, J. Partial Differ. Equ. 29, 102-115 (2016)

10. Cao, D.M.: Nontrivial solution of semilinear elliptic equation with critical exponent in $\mathbb{R}^{2}$. Commun. Partial Differ. Equ. 17, 407-435 (1992)

11. Chen, Y., McKenna, P.J.: Traveling waves in a nonlinear suspension beam: theoretical results and numerical observations. J. Differ. Equ. 135, 325-355 (1997)

12. do Ó, J.M.B.: Quasilinear elliptic equations with exponential nonlinearities. Commun. Appl. Nonlinear Anal. 2, 63-72 (1995)

13. do Ó, J.M.B.: $N$-Laplacian equations in $R^{N}$ with critical growth. Abstract Appl. Anal. 2, 301-315 (1997)

14. Ekeland, I.: On the variational principle. J. Math. Anal. App. 47, 324-353 (1974)

15. Ferrero, A., Gazzola, F.: A partially hinged rectangular plate as a model for suspension bridges. Discrete Contin. Dyn. Syst. 35, 5879-5908 (2015)

16. Gazzola, F., Grunau, H., Sweers, G.: Polyharmonic Boundary Value Problems. Lectures Notes in Mathematics. Springer, Berlin (2010)

17. Lam, N., Lu, G.: Sharp singular Adams inequalities in high order Sobolev Spaces. Methods Appl. Anal. 19, 243-266 (2012)

18. Lam, N., Lu, G.: Existence of nontrivial solutions to polyharmonic equations with subcritical and critical exponential growth. Discrete Contin. Dyn. Syst. 32, 2187-2205 (2012)

19. Lam, N., Lu, G.: A new approach to sharp Moser-Trudinger and Adams type inequalities: a rearrangementfree argument. J. Differ. Equ. 255, 298-325 (2013)

20. Lam, N., Lu, G., Bao, J.: Polyharmonic equations with critical exponential growth in the whole space $\mathbb{R}^{n}$. Discrete Contin. Dyn. Syst. 36, 577-600 (2016) 
21. Lazer, A.C., McKenna, P.J.: Large-amplitude periodic oscillations in suspension bridges: some new connections with nonlinear analysis. SIAM Rev. 225, 352-370 (2005)

22. Li, Y., Ruf, B.: A sharp Trudinger-Moser type inequality for unbounded domains in $\mathbb{R}^{n}$. Indiana Univ. Math. J. 57, 451-480 (2008)

23. Lu, G., Yang, Y.: Adams' inequalities for bi-Laplacian and extremal functions in dimension four. Adv. Math. 220, 1135-1170 (2009)

24. Masmoudi, N., Sani, F.: Adams' inequality with the exact growth condition in $\mathbb{R}^{4}$. Commun. Pure Appl. Math. 67, 1307-1335 (2014)

25. Moser, J.: A sharp form of an inequality by N. Trudinger. Indiana Univ. Math. J. 20, 1077-1092 (1971)

26. Ozawa, T.: On critical cases of Sobolev's inequalities. J. Funct. Anal. 127, 259-269 (1995)

27. Pimenta, M.T.O., Soares, S.H.M.: Existence and concentration of solutions for a class of biharmonic equations. J. Math. Anal. Appl. 390, 274-289 (2012)

28. Ruf, B.: A sharp Trudinger-Moser type inequality for unbounded domains in $\mathbb{R}^{2}$. J. Funct. Anal. 219, 340-367 (2005)

29. Ruf, B., Sani, F.: Sharp Adams-type inequalities in $\mathbb{R}^{N}$. Trans. Am. Math. Soc. 365, 645-670 (2013)

30. Sani, F.: A biharmonic equation in $\mathbb{R}^{4}$ involving nonlinearities with subcritical exponential growth. Adv. Nonlinear Stud. 11, 889-904 (2011)

31. Sani, F.: A biharmonic equation in $\mathbb{R}^{4}$ involving nonlinearities with critical exponential growth. Commun. Pure Appl. Anal. 12, 405-428 (2013)

32. Strauss, W.A.: Existence of solitary waves in higher dimensions. Commun. Math. Phys. 55, 149-162 (1977)

33. Su, J., Wang, Z.-Q., Willem, M.: Nonlinear Schrödinger equations with unbounded and decaying radial potentials. Commun. Contemp. Math. 9, 571-583 (2007)

34. Su, J., Wang, Z.-Q., Willem, M.: Weighted Sobolev embedding with unbounded and decaying radial potentials. J. Differ. Equ. 238, 201-219 (2007)

35. Tarsi, C.: Adams' inequality and limiting Sobolev embeddings into Zygmund spaces. Potential Anal. 37, 353-385 (2012)

36. Trudinger, N.S.: On the embedding into Orlicz spaces and some applications. J. Math. Mech. 17, 473-484 (1967)

37. Yang, Y.: Adams type inequalities and related elliptic partial differential equations in dimension four. J. Differ. Equ. 252, 2266-2295 (2012)

38. Ye, Y.W., Tang, C.L.: Infinitely many solutions for fourth-order elliptic equations. J. Math. Anal. Appl. 394, 841-854 (2012)

39. Ye, Y., Tang, C.: Existence and multiplicity of solutions for fourth-order elliptic equations in $\mathbb{R}^{N}$. J. Math. Anal. Appl. 406, 335-351 (2013)

Publisher's Note Springer Nature remains neutral with regard to jurisdictional claims in published maps and institutional affiliations. 